Z. klin. Chem. u. klin. Biochem.

8. Jg., S. 49-50, Januar 1970

\title{
Quantitative Bestimmung von Furosemid und seines Metaboliten in Harn mit Hilfe der Sephadex-Säulenchromatographie
}

\author{
Von P. Hajdú und I. Hornke \\ Farbwerke Hoechst AG, vormals Meister Lucius \& Brüning
}

(Eingegangen am 20. August 1969)

Es wird über die säulenchromatographische Trennung an Sephadex G 25 als weiteres Nachweisverfahren zur Untersuchung der Ausscheidungsprodukte und -mengen des Salidiureticums Furosemid (Lasix) in Ham berichtet.

Mit diesem einfachen Verfahren erreicht man eine Nachweisgrenze von maximal $0,5 \mu \mathrm{g} / \mathrm{m} /$ für beide Substanzen bei Verwendung von UV-Durchflußmikroküvetten.

The quantitative determination of furosemide and its metabolites in urine with the aid of Sephadex column chromatograpby Column chromatography on Sephadex G 25 was used to study further the nature and quantity of the urinary excretory products of the salidiuretic furosemide (Lasix).

With this simple method, the detection limit for both substances was $0.5 \mu \mathrm{g} / \mathrm{ml}$, with the use UV-flow-through microcuvettes.

In früheren Arbeiten unseres Laboratoriums $(1,2,3)$, wurde eingehend über Nachweismethoden von 4-Chlor$\mathrm{N}$-(2-furylmethyl)-5-sulfamoyl-anthranilsäure (Furosemid) ${ }^{1}$ ) in Körperflüssigkeiten sowie über die papierchromatographische Trennung des Metaboliten 4-Chlor5-sulfamoyl-anthranilsäure berichtet. In einer dieser Veröffentlichungen (2) ist eine kolorimetrische Methode beschrieben worden, mit der der kinetische Verlauf der Metabolisierung an Hunden (nach Gabe von 5-50 $\mathrm{mg} / \mathrm{kg}$ ) verfolgt werden konnte.

Die Untersuchung von Humanharn-Proben nach Verabreichung von 1-2 Tabletten (à $40 \mathrm{mg}$ ) oder Ampullen (à $20 \mathrm{mg}$ ), also $\leqq 1 \mathrm{mg} / \mathrm{kg}$ brachte methodische Schwierigkeiten (4). Um diese auszuschließen und die Kinetik der Metabolisierung auch an Menschen prüfen zu können, wurde von uns ein neues, für den Routine-Betrieb geeignetes Verfahren ausgearbeitet.

Es werden hierbei Humanharnextrakte (vgl. (2)) durch Säulenchromatographie an Sephadex G 25 mit NaClLösung als Elutionsmittel getrennt und die Lichtdurchlässigkeit des Eluats bei $233 \mathrm{~nm}$ kontinuierlich aufgezeichnet. Die Harnkonzentrationen beider Verbindungen können aus den Flächen der aufgezeichneten Kurven errechnet werden.

\section{Methodik}

$5 \mathrm{ml}$ einer Harnprobe werden nach Ansäuern $(0,25 \mathrm{ml} \mathrm{konz} . \mathrm{HCl})$ mit $50 \mathrm{ml}$ Äther ausgeschüttelt. Die abgetrennte wäßr. Phase. versetzt man nochmals mit $50 \mathrm{~m} /$ Äther und fügt unter Schütteln so lange wasserfreies $\mathrm{Na}_{2} \mathrm{SO}_{4}$ hinzu, bis die wäßr. Phase als Kristallwasser gebunden ist und das Trockenmittel im festen Zustand vorliegt. Anschließend wird die Ätherphase in einen Kolben filtriert und $2 \mathrm{mal} \mathrm{mit} 5 \mathrm{ml}$ Ather nachgespült. Die vereinigten Âtherauszüge werden dann am Rotationsverdampfer zur Trockene gebracht und der Rückstand mit 0,9proz. NaCl-Lösung aufgenommen. In der vorliegenden Arbeit wurden sowohl die wäßr. Eichwerte als auch die Versuche in Harn mit NaCl-Lösung auf $5 \mathrm{ml}$, das Ausgangsvolumen, aufgefüllt.

Zahlreiche Versuche zeigten, daß die Trennung ebenso sauber und die Kurvenfäche quantitativ auszuwerten ist, wenn die Trocken-

1) = Lasix, Farbwerke Hoechst AG. rückstānde mit weniger $\mathrm{NaCl}$-Lösung (z. B. $1,25 \mathrm{~m} /$ ) aufgenommen werden. Somit kann eine bis zu $4 \mathrm{mal}$ höhere Nachweisgrenze $(0,5 \mu \mathrm{g} / \mathrm{m} / \mathrm{Harn})$ erreicht werden.

$1 \mathrm{ml}$ des Harnextraktes wird auf eine Sephadex G 25-Säule (Säulendurchmesser $1,5 \mathrm{~cm}$, Säulenhöhe $30 \mathrm{~cm}$; $9 \mathrm{~g}$ Sephadex G 25 werden in $100 \mathrm{ml} 0,9$ proz. $\mathrm{NaCl}$-Lösung gequollen) aufgetragen und mit physiol. NaCl-Lösung in diffusem Licht eluiert. Die Lichtdurchlässigkeit des Eluats wurde mit Hilfe der Mikrodurchflußküvette MR 1D in einem Zeiss PMQ II Spektralphotometer mit Mikroküvettenausrüstung kontinuierlich gemessen und mit einem Philips Kompensationsschreiber (0-100\% Lichtdurchlässigkeit $=20 \mathrm{~cm}$, Papiervorschub $1 \mathrm{~mm} / \mathrm{Min}$.) aufgezeichnet.

\section{Ergebnisse und Diskussion}

Wie aus Abbildung 1 ersichtlich, werden zunächst die bei der angegebenen Wellenlänge absorbierenden Kom-

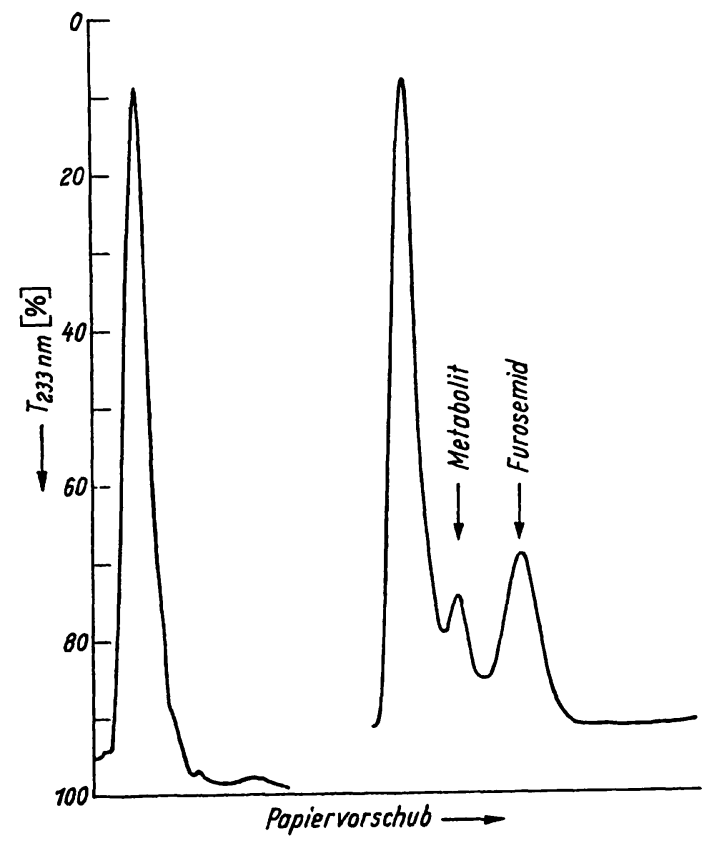

Abb. 1

Trennung von Furosemid und seines Metaboliten in Harn an einer Sephadex G25 Säule. Links: Leerharn; rechits: Harn mit Furosemid und seinem Metaboliten 4-Chlor-5-sulfamoyl-anthranilsäure versetzt 


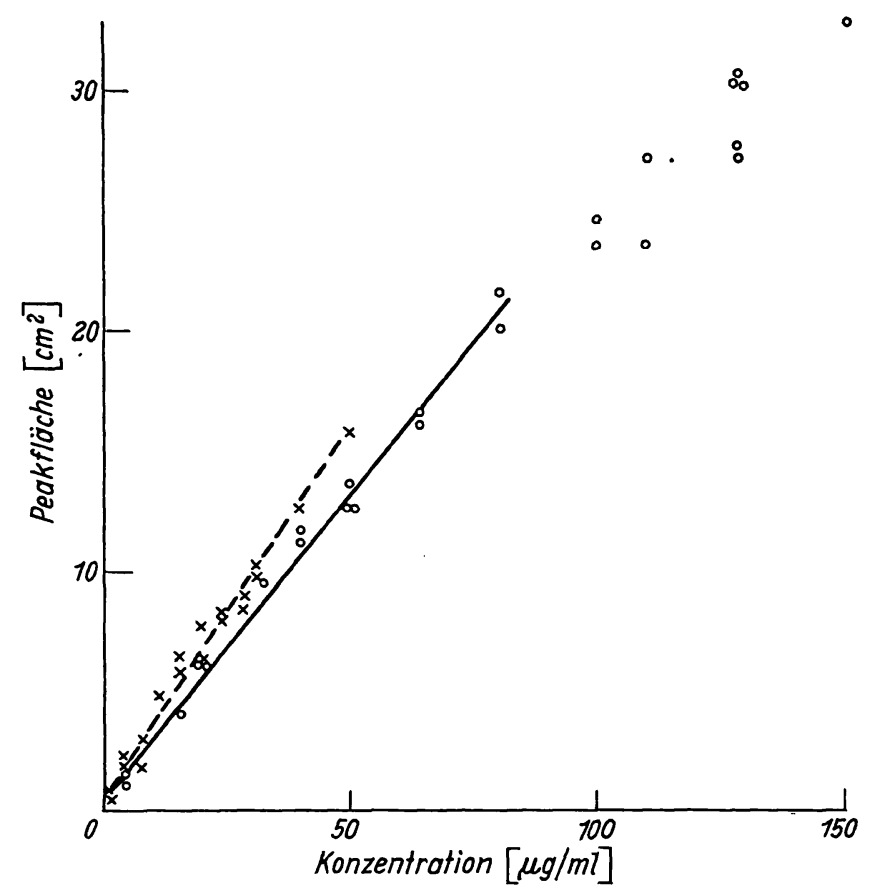

Abb. 2

Eichwerte wäßr. Lösungen von Furosemid $(\circ-0)$ und 4-Chlor-5sulfamoyl-anthranilsäure $(x-x)$ als Peakflächen des Elutionsdiagramms $\left(T_{33 s} \mathrm{~nm}\right)$

ponenten des Urins von der Säule eluiert, denen dann der Metabolit und schließlich das Furosemid folgen.

Es fällt hierbei auf, daß das Furosemid nicht, wie bei der Gelfiltration zu erwarten, vor der 4-Chlor-5-sulfamoylanthranilsäure erscheint, sondern in umgekehrter Reihenfolge. Eine derartige Abweichung von den Regeln der Gelfiltration läßt den Schluß zu, daß es sich im vorliegenden Fall um eine reine adsorptionschromatographische Trennung an Sephadex handelt. Derartige Abweichungen von dem Prinzip der Gelfiltration an Sephadex konnten schon früher beobachtet werden $(5,6)$.

Wäßr. Lösungsgemische mit verschiedenen Furosemid(4-150 $\mu \mathrm{g} / \mathrm{ml})$ und 4-Chlor-5-sulfamoyl-anthranilsäure$(1-50 \mu \mathrm{g} / \mathrm{m} /)$ Konzentrationen wurden angesetzt und wie oben beschrieben analysiert.

Die erhaltenen Ergebnisse - aus Abbildung 2 ersichtlich - zeigen, daß neben einer guten Trennung beider Verbindungen der lineare Zusammenhang zwischen den Flächen und den Konzentrationen (bis zu 80 bzw. 50 $\mu \mathrm{g} / \mathrm{m} l$ ) eine genaue (mittlerer Fehler $\pm 2,4$ bzw. $1,8 \mu \mathrm{g} / \mathrm{m} l$ Extrakt) Möglichkeit zur quantitativen Er- fassung von Furosemid und seines Metaboliten nebeneinander bietet.

Die Tabelle stellt die Anyalsenergebnisse von HumanHarn, dem bekannte Mengen Furosemid bzw. seines Metaboliten zugefügt worden waren, dar. Die Konžentrationen wurden aus Eichgeraden, die für wäßr. Lösungen erstellt worden waren, errechnet. Die wiedergefundenen Substanzmengen zeigen, da $\beta$ mit dieser Trenn- und Bestimmungsmethode eine Empfindlichkeit sowohl für den Metaboliten als auch für Furosemid von $1,5-2 \mu \mathrm{g} / \mathrm{m} l$ Extrakt erreicht werden $\mathrm{kann}^{2}$ ).

Diese Methode eröffnet durch ihre einfache und schnelle Durchführung die Möglichkeit größerer Kontrolluntersuchungen über die Ausscheidungsrate von Furosemid sowie dessen Metabolisierung im menschlichen Körper.

Tab. 1

Säulenchrömatographische Analyse von Furosemid und seines Metaboliten in Harn (Mehrfachbestimmungen jeweils zugesetżter Sub-
stanz)

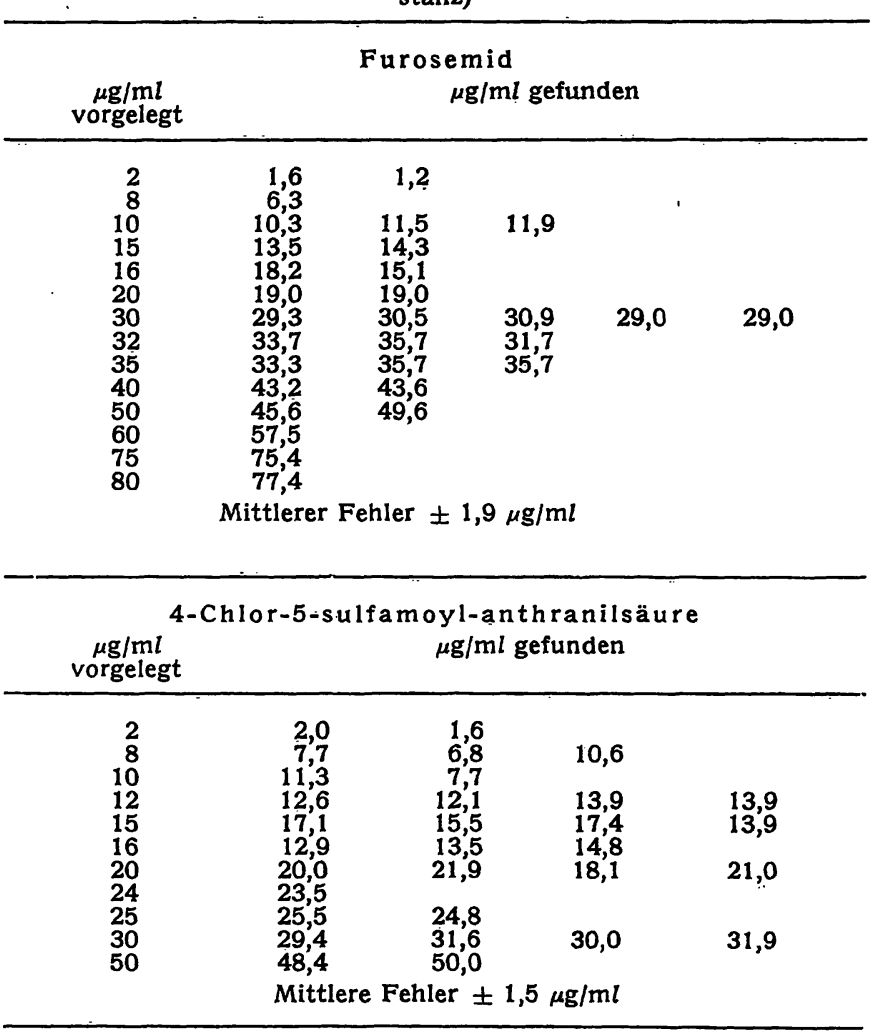

2) Verwendet man ein Durchflußfluorometer, so kann die Empfindlichkeit noch weiter gesteigert werden.

\section{Literatur}

1. HajDú, P. und A. Häussler, Atzneimittel-Forsch. Aulendorf 14, 709 (1964). - 2. Häussler, A. und P. HajDú, ArzneimittelForsch. Aulendorf 14, 710 (1964). - 3. HäussLER, A. und H. Wrcha, Arzneimițtel-Forșch. Aụlendorf 15, 81 (1965). - 4.
BürtNER, H., Persönliche Mitteilung. - 5. ,Sephadex: Eine kurze Einführung in das Verfahren der Gel-Filtration". Pharmacia, Uppsala Schweden. - 6. Gerotre, B., J. Chromatog. 3, 330 (1960).

Dipl.-Chem. P. Hajdú Farbwerke Hoechst 6230 Frankfurt/M.-Hoechst Physiolog.-Chem. Labor D 528 